40 Il faut employer la méthode de Frost avec une température de $27^{\circ}$ et un séjour à l'étuve de 20 à 24 heures.

$5^{\circ}$ Les échantillons doivent être dilués au préalable, au cas échéant, avec une solution de peptone + chlorure de sodium, soit au 1/10, soit au $1 / 100$.

$$
*^{*} *
$$

Ainsi que le fait remarquer mon collègue, M. le professeurvan OIJEN, qui a bien voulu m'adresser ce travail, les expérimentateurs Frost et Clarenburg ont ainsi introduit dans notre arsenal analytique microbien, une méthode élégante et précise, dont doivent leur être obligés tous ceux qui sont chargés du contrôle hygiénique du lait.

\title{
LA COMPOSITION CHIMIQUE DE LA GLANDE IMAMIMAIRE DE LA VACHE
}

\author{
par le professeur Dr Otakar LAXA.
}

Nos connaissanses sur la composition chimique de la glande mammaire sont, jusqu'à nos jours, restées insuffisantes. Par exemple, nous chercherions en vain les documents analytiques qui illustrent la composition chimique la plus simple de la glande mammaire. Et pourtant, il semble qu'on devrait en tirer quelque chose pour l'explication de la formation du lait.

TABLEAU 1

\begin{tabular}{|c|c|c|c|c|c|c|c|c|c|c|}
\hline & 1 & 2 & 3 & 4 & 5 & 6 & 7 & 8 & 9 & Moyen \\
\hline Eau $\ldots \ldots \ldots$ & 73,79 & 78,99 & 79,96 & 76,12 & 78,06 & 78,98 & 75,28 & 72,73 & 73,70 & $76,4 \mathrm{C}$ \\
\hline Matières grasses ... & 12,31 & 5,62 & 4,97 & 8,16 & 7,50 & 8,62 & 10,47 & 14,13 & 13,77 & $9,5 \mathrm{C}$ \\
\hline Matières azotées.... & 11,04 & 11,61 & 14,50 & 14,63 & 13,01 & 9,79 & 10,87 & 11,70 & 9,38 & 11,84 \\
\hline Cendras... & 1,45 & 1,46 & 1,28 & 0,95 & 1,03 & 1,24 & 1,38 & 0,90 & 0,98 & 1,18 \\
\hline Matières indosées... & 1,41 & 2,32 & 0 & 0,14 & $C, 40$ & 1,37 & 2,00 & 0,54 & 2,17 & $1,0 \varepsilon$ \\
\hline Matière sèche. & 26,21 & 22,01 & 20,04 & 83,88 & 21,94 & 21,02 & 24,72 & 27,27 & 26,30 & 23,60 \\
\hline
\end{tabular}


J'ai fait quelques analyses de la glande mammaire sur des pièees d'origine inconnue. Dans tous les eas, j'ai choisi la masse glandulaire compacte, débarrassée des gros vaisseaux et des enveloppes conjonctives. La masse était broyée dans un hâchoir à viande et on en a dosé le contenu de l'eau par séchage sur sable siliceux dans une étuve à $100^{\circ} \mathrm{C}$. Dans le résidu sec, on a dosé la graisse par l'extraction à l'éther. Les matières azotées étaient calculées après dosage d'azote, déterminé par le procédé de KJELDAHL, en utilisant le facteur 6,37. Les cendres étaient obtenues par la calcination dans une capsule de platine. Les matières non dosées directement étaient calculées par différence. Le tableau 1 donne les résultats en \% sur de la glande fraîche; le tableau 2 montre les pourcentages dans la matière sèche et le tableau 3 contient les mêmes pourcentages sur la matière sèche dégraissée.

TABLEAU 2

\begin{tabular}{|c|c|c|c|c|c|c|c|c|c|c|}
\hline & 1 & 2 & 3 & 4 & 5 & 6 & 7 & 8 & 9 & Iloyen \\
\hline Matières grasses. & 46,96 & 26,70 & 24,50 & 34,17 & 34,18 & 41,09 & 42,35 & 51,81 & 52,35 & 39,32 \\
\hline Matières azotées.... & 42,12 & 55,20 & 72,50 & 61,44 & 59,29 & 46,57 & 43,97 & 42,90 & 35,66 & 51,07 \\
\hline Cendres. & 5,53 & 6,40 & 6,40 & 3,98 & 3,51 & 5,89 & 5,58 & 3,30 & \begin{tabular}{l|l|}
0 & 3,73 \\
\end{tabular} & 4,92 \\
\hline Matières indosées ... & 5,39 & 11,70 & 0 & 0,59 & 1,84 & 6,45 & 8,10 & 1,98 & 8,80 & 4,98 \\
\hline
\end{tabular}

TABLEAU 3

\begin{tabular}{|c|c|c|c|c|c|c|c|c|c|c|}
\hline & 1 & 2 & 3 & 4 & 5 & 6 & 7 & -8 & 9 & Moyen \\
\hline Matières azotées.... & 79,3 & 69,9 & 90,2 & 93,08 & 90,09 & 78,94 & $7.6,28$ & 89,04 & 74,86 & 82,41 \\
\hline Cendres ..... & 10,4 & 8,8 & 9,8 & 6,0 & 7,01 & 10,00 & 9,68 & 6,85 & 7,82 & 8,48 \\
\hline Matières indosées & 10,3 & 21,3 & 0 & 0,88 & 2,90 & 11,06 & 14,04 & 4,11 & 17,32 & 9,11 \\
\hline
\end{tabular}

D'après ces analyses, il est évident que la composition de la glande mammaire ne varie que dans les limites assez étroites. Les différences 
sont produites surtout par le contenu en graisse. On peut distinguer des glandes qui contiennent plus de graisse que d'albuminoïdes: et celles dans lesquelles les protéines sont en plus grande quantité que la graisse. Les échantillons 1, 8, 9 appartiennent à des glandes très grasses, la graisse $y$ est en plus grande proportion que les albuminoïdes; dans d'autres cas, la glande est plus riche en protéines, qui sont le siège des phénomènes essentiels de la sécrétion.

On peut considérer que les glandes de la première espèce, les très grasses, appartenaient à des vaches en fin de lactation, destinées à la boucherie, donc des vaches engraissées.

Eau : Le contenu de l'eau varie de 72,73 à 78,99\%; en moyenne, il est de $76,40 \%$.

Matière sèche : Elle oscille entre 20,04 à $27,27 \%$, elle est en moyenne $23,6 \%$, soit deux fois plus que dans le lait.

Matières grasses: Ce sont elles qui varient dans la plus grande mesure, de 4,97 à $14,13 \%$ dans la glande fraîche, de 24,5 à $52,35 \%$ dans le résidu sec. Il est intéressant de noter que le minimum, $(24,5 \%)$ correspond à celui qu'on remarque dans le lait de vache.

Matières azotées: Calculées comme protéines, elles ne sont pas soumises à des oseillations aussi grandes que la graisse, si on considère les chiffres $9,79,-14,63 \%$ dans la glande fraîche; mais les chiffres 'sont nettement discordants dans la matière sèche : 23,37 à $72,50 \%$.

Cendres: Les pourcentages sont de 0,9 à 1,46 dans la glande fraiche, 3,51 à $6,4 \%$ dans la matière sèche.

Les matières indosées ont accusé les chiffres 0 à $2,32 \%$ dans la glande fraîche et 0 à $8,80 \%$ dans la matière sèche, done la quantité en est petite.

Mais il y a lieu maintenant d'examiner des composés chimiques du tissu de la glande.

La graisse :La graisse de la glande mammaire est d'une couleur jaune vif qui disparaît quand on la chauffe quelques heures à $100^{\circ} \mathrm{C}$.

La graisse de la glande mammaire extrait par l'éther de la substance desséchée à $70^{\circ} \mathrm{C}$. et qui avait été lavée à l'eau bouillante a donné les constantes suivantes:

\begin{tabular}{|c|c|c|}
\hline dice & on & $196,5-197,1$ \\
\hline$n$ & de Reichert.Meissl & 6,82 \\
\hline ) & de Wauters-Polenske ....... & 1,5 \\
\hline ) & de réfraction à $40^{\circ} \mathrm{C} \ldots \ldots \ldots \ldots$ & 48 \\
\hline " & d'iode $\ldots \ldots \ldots \ldots \ldots \ldots \ldots \ldots \ldots \ldots$ & $48,23-48,1$ \\
\hline D & des acides libres & 36 \\
\hline n & de & 94,9 \\
\hline ids & isolubles & 286. \\
\hline
\end{tabular}

La graisse de la même glande mammaire qui n'avait pas été lavée à l'eau bouillante, a donné des constantes un peu différentes: 
Indice de la saponification

$211,2-210,8$

" de Reichert-Meissl

1,2

(3)

$41,8-42,3$

Poids moléculaire des acides insolubles

298,6

La graisse de la glande mammaire diffère de la graisse du lait par la petite quantité des acides volatiles et cette différence est attribuée, dans une certaine mesure, à la graisse de dépôt que retirent le tissu de la masse glandaire. Les earactères de la graisse de la glande mammaire la rapproche du saindoux, d'autant plus que la lactation est sur sa fin et que la vache se trouve "sèche ". L'indice de saponification diminue dans ces cas jusqu'aux chiffres 198-200.

Il est singulier que la graisse de la glande mammaire, dans tous les cas montre une grande acidité, c'est-à-dire qu'elle contient des acides gras libres. L'indice des acides libres oscille entre 8-36. Cette grande acidité de la graisse est d'autant plus intéressante que la graisse du lait est presque neutre. Probablement, dans la glande, une lipase est-elle en action, qui met en liberté les acides gras des phosphatides, décompose la graisse déposée dans les cellules du tissu, et e'est avec ces produits de décomposition que la graisse du lait serait ultérieurement élaborée.

TABLEAU 5 (glande fraîche)

\begin{tabular}{|c|c|c|c|c|c|c||}
\hline \hline Echantillon & 3 & 5 & 6 & 7 & 8 & moyen \\
\hline Eau ........ & 79,96 & 78,06 & 78,98 & 75,28 & 72,73 & 77,00 \\
\hline Graisse ....... & 4,07 & 6,51 & 6,85 & 9,24 & 12,08 & 7,61 \\
\hline Cholesterine .... & non dosé & 0,22 & 0,77 & 0,37 & 0,72 & 0,52 \\
\hline Phosphatides ... & 0,90 & 0,77 & 1,00 & 0,86 & 1,33 & 0,97 \\
\hline Matières azotées. & 14,50 & 13,01 & 9,79 & 10,87 & 11,70 & 11,97 \\
\hline & & & & & & \\
\hline Matières indosées & 0 & 0,40 & 1,37 & 2,00 & 0,54 & 1,06 \\
\hline
\end{tabular}


Dans le résidu sec :

\begin{tabular}{|r|r|r|r|r|r|r|}
\hline \hline Graisse ....... & 19,34 & 29,67 & 32,59 & 37,37 & 44,29 & 32,25 \\
\hline Cholestérine .... & non dosé & 1,00 & 3,66 & 1,49 & 2,63 & 2,19 \\
\hline Phosphatides ... & 4,28 & 3,51 & 4,76 & 3,48 & 4,87 & 4,18 \\
\hline Matières azotées. & 72,50 & 59,29 & 46,57 & 43,97 & 42,90 & 53,04 \\
\hline Matières indosées & & 1,84 & 6,45 & 8,10 & 1,98 & 4,59 \\
\hline Cendres ....... & 6,40 & 3,51 & 5,89 & 5,58 & 3,30 & 4,93 \\
\hline
\end{tabular}

Phosphatides et Stérines: Ainsi que je l'avançais il y a déjà 14 ans, (1) la glande mammaire est riche en phosphatides et en cholestérine. J'ai dosé ces deux lipoïdes dans quelques échantillons et les résultats obtenus ont été donnés dans le tableau 5.

On voit que la cholestérine forme 1-3,66 \% de la matière sèche, et dans la graisse $3,3-11 \%$ tandis que dans la graisse du lait, le contenu de cholestérine ne depasse pas $0,5 \%$.

Même dans le sang de chèvre, ABDERHALDEN n'a trouvé que $0,65 \%$ de cholestérine dans le résidu sec.

On rencontre aussi les phosphatides en plus grande quantité. Les chiffres 3,48-4,87\% dans la matière sèche font dans la graisse $11-14,5 \%$, solt une quantité considérable, si on sait qu'il n'y a que $0,01 \%$ de phosphatides dans la graisse du lait. Dans le sang de chèvre, ABderHaLden a décelé $1,23 \%$ des phosphatides dans le résidu sec. Pourquoi la nature concentre-t-elle ces matières dans la glande mammaire et n'en laisse passer que des traces dans le lait? Le grand rôle des phosphatides dans la formation du lait est par cela évident. Pourrait-on les ranger dans les précurseurs de la graisse dans le lait ?

Matières azotées: Un quart jusqu'à trois quarts de la matière sèche de la glande est composé de matières azotées, généralement des albuminoïdes. Quand on prépare un extrait à l'eau froide de la glande fraîche, on obtient un liquide louche qui ne précipite pas par les acides, mais qui se trouble plus fortement par l'ébullition et précipite par le tanin. Le dosage des diverses formes des matières azotées dans un échantillon de la glande fraîche a donné les résultats suivants:

(1) Milchwirtschaftliches Zentralblatt, 1913, 42. 663. 


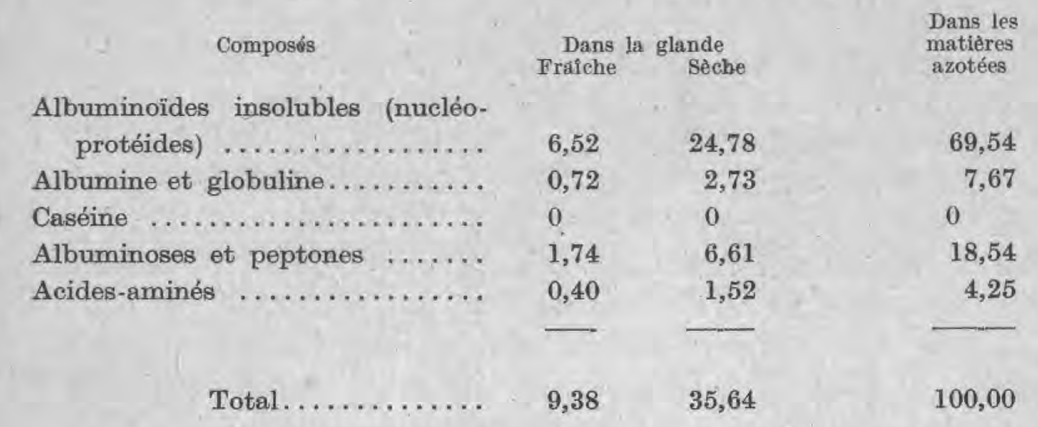

La majeure partie des matières protéiques est composée de nucléoprotéides insolubles dans l'eau. Ces matières sont insolubles dans la solution d'oxalate de sodium. Cette liqueur d'ailleurs ne dissout rien de la glande fraîche. La présence des albuminoïdes etdes peptones. des acides-aminés dans la glande fraîche répond à la théorie de HnDEBRAND sur l'origine de la caséine, théorie qui fait intervenir un ferment protéolytique de la glande, lequel décomposerait d'abord les albuminoïdes du sang et constituerait ensuite la caséine avec les produits de cette autolyse. Remarquons que cette hypothèse de MANDEL n'exclut pas celle selon laquelle la caséine prendrait naissance aux dépens des produits de décomposition des nucléoprotéides de la glande.

Cendres : Elles sont riches en chaux et en acide phosphorique. J'ai trouvé dans les cendres $18-24 \%$ de chaux et $32 \%$ d'acide phosphorique, done une quantité correspondant presque à la quantité de ces composés dans le lait.

Les matières indosées: La qualité des matières non identifiées n'est pas uniforme. On peut soupçonner la présence du sucre du lait et de l'acide lactique. En vérité, la grande partie de ces substances est formée de sucre du lait. J'ai fait chauffer du tissu de la glande mammaire dans l'eau à $100^{\circ} \mathrm{C}$. pendant 1 heure. Le liquide séparé de la masse coagulée a été précipité par le tanin et on a filtré. Dans le liquide clair et incoloré, on a dosé le résidu sec, le lactose par la réduction de la liqueur de FEHLING, et l'acide lactique par le calcul de l'acidité de la glande fraîche, les cendres et les matières azotées non protéiques.Voici les résultats :

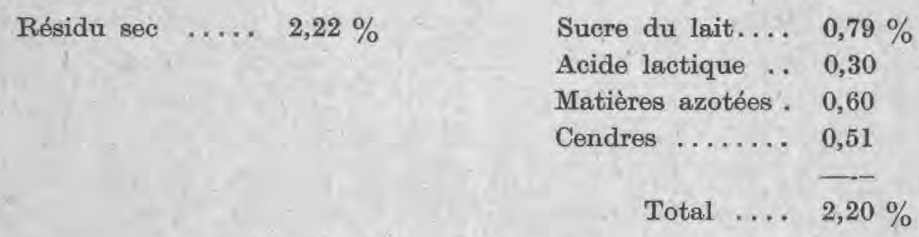

Il ne reste donc presque rien qui puisse correspondre à des matières de earactère inconnu. Il s'agit principalement de me persuader dans 
quelle mesure on peut juger de la présence de la gomme animale dans la glande mammaire de la vache. LANDWEHR a isolé cette substance de la glande mammaire des lapins et il l'a qualifié comme substance mère du sucre du lait. Pour trouver cette substance dans la glande de la vache, j'ai choisi l'extrait aqueux de la glande, préparé par le procédé ci-dessus cité, dépourvu d'albuminoïdes. J'ai évaporé ce liquide au bain-marie après l'addition d'acide chlorhydrique jusqu'à consistance assez épaisse, tandis que le sucre du lait était décomposé en glucose et galactose, e'est-à-dire en sucres solubles dans l'alcool. Ce liquide était mélangé dans un petit ballon avee l'alcool. Après 24 heures il s'est déposé sur les parois un faible précipité cristallin de chlorure de sodium. Je n'ai done pas décelé des substances gommeuses. Le cas observé par LANDWEHR est donc tout spécial et les matières gommeuses observées par lui ne sont pas en liaison avec l'origine du sucre du lait.

\title{
QUELQUES TRAVAUX DE L'OFFICE AGRICOLE DE SEINE-ET-OISE
}

\author{
(suite)
}

par M. MONMIREL,

Président de l'Office agricole départemental de Seine-et-Oise

\section{III. - DÉTERMINATION DU PRIX DE REVIENT DE L'EN- SILAGE. - PRIX DE REVIENT COMPARÉS DES BETTERAVES $1 / 2$ SUGRIERES ET DE L'ENSILAGE DE MAİS.}

Au cours de l'été 1925 des essais ont été entrepris dans le but de déterminer, aussi exactement que possible, le prix de revient de l'ensilage en silo métallique. L'on a utilisé pour cela le silo de la ferme de la Faisanderie, qui venait d'être rempli, avantla moisson, d'un mélange de vesee, d'avoine et de poix fourragers. Un stagiaire, ancien élève de l'école d'Agriculture de Grignon, M. SHTEIGELBERg a séjourné à Villepreux du 29 juin au 5 juillet pour étudier les conditions de la récolte $d u$ fourrage et du remplissage du silo. L'ensilage obtenu a été mis en consommation à partir du 21 août. Deux jours avant cette date, M. Duvivier, ancien élève de l'Ecole d'Agriculture de Montpellier, s'est rendu à son tour sur l'exploitation dans le but d'assister à la vidange du silo et de déterminer le poids de fourrage consommé par les animaux, ainsi que le rapport de ce poids à celui de la matière verte initiale. Il a également suivi attentivement les opérations de remplissage du silo avec du maïs fourrage, qui eurent lieu du 21 au 30 septembre.

De nombreux prélèvements, effectués par les soins des collaborateurs précités, ant été envoyés au laboratoire de chimie analytique 